This item was submitted to Loughborough's Research Repository by the author.

Items in Figshare are protected by copyright, with all rights reserved, unless otherwise indicated.

\title{
Investigation of whisker growth from alkaline non-cyanide zinc electrodeposits
}

PLEASE CITE THE PUBLISHED VERSION

http://dx.doi.org/10.1007/s11664-016-5074-4

\section{PUBLISHER}

(C) The Author(s). This article is published with open access at Springerlink.com

\section{VERSION}

VoR (Version of Record)

\section{PUBLISHER STATEMENT}

This work is made available according to the conditions of the Creative Commons Attribution 4.0 International (CC BY 4.0) licence. Full details of this licence are available at: http://creativecommons.org/licenses/ by/4.0/

\section{LICENCE}

CC BY-NC-ND 4.0

\section{REPOSITORY RECORD}

Wu, L., Mark A. Ashworth, and G.D. Wilcox. 2019. "Investigation of Whisker Growth from Alkaline Non-cyanide Zinc Electrodeposits". figshare. https://hdl.handle.net/2134/23312. 


\title{
Investigation of Whisker Growth from Alkaline Non-cyanide Zinc Electrodeposits
}

\author{
L. WU, ${ }^{1,2}$ M.A. ASHWORTH, ${ }^{1}$ and G.D. WILCOX ${ }^{1}$ \\ 1.-Department of Materials, Loughborough University, Loughborough, Leicestershire LE11 3TU, \\ UK. 2.—e-mail: l.wu@lboro.ac.uk
}

Electroplated zinc finishes have been widely used in the packaging of electronic products for many years as a result of their excellent corrosion resistance and relatively low cost. However, the spontaneous formation of whiskers on zinc electroplated components, which are capable of resulting in electrical shorting or other damaging effects, can be highly problematic for the reliability of long-life electrical and electronic equipment. This work investigated the mechanism for whisker growth from zinc electrodeposited mild steel substrates. The incubation time for whisker growth from the surface of nodules on the surface of the electrodeposit was considerably reduced compared with that from the planar deposit surface. Recrystallisation of the as-deposited columnar structure was observed at the whisker root. This result is consistent with some recent whisker growth models based on recrystallisation. There was no evidence of iron-zinc (Fe-Zn) intermetallic formation at the iron/zinc $(\mathrm{Fe} / \mathrm{Zn})$ interface or within the zinc coating beneath the whiskers.

Key words: Whiskers, zinc, electrodeposition, mechanisms

\section{INTRODUCTION}

Metallic whiskers are spontaneous growths that most often develop from metal surfaces such as tin, zinc and cadmium, in particular when they are used in the form of electroplated coatings. Normally, metallic whisker growth undergoes an uncertain incubation period ranging from hours to years, followed by a highly variable growth rate. ${ }^{1}$ The growth of metallic whiskers is capable of inducing localised short circuiting and is problematic for the reliability of long-life electrical and electronic components. Failures caused by whisker growth have been reported in telecommunications-, military- and aerospace-based applications, with consequences ranging from mild inconvenience to complete system failures. ${ }^{1}$ The study of metallic whiskers can be traced back to the $1940 \mathrm{~s}$, when the presence of cadmium whiskers resulted in the breakdown of radio systems used in aircraft during World War II. ${ }^{2}$ Over the past 70 years, the majority of research effort has been focused on tin whiskers, and today

(Received November 18, 2015; accepted October 18, 2016) whisker growth on tin is a well-documented phenomenon; in comparison, much less attention has been paid to the investigation of zinc whiskers. Zinc finishes have been widely used for many years and are normally electroplated on ferrous components as a corrosion-resistant coating. For example, the steel sheet metal on raised floor tiles used in computer data centres is sometimes protected with electroplated zinc coatings. However, filament-type zinc whiskers have been found on the bottom surface of such floor tiles and are prone to break off and become airborne. These whiskers can be carried in the turbulent air flow and drawn into computer equipment by cooling fans and finally cause electrical failures. During the last two decades, a number of computer equipment failures have been attributed to problems induced by zinc whisker growth. ${ }^{3,4}$

In the public literature, very little effort has been paid to investigate zinc whisker growth. A number of mechanisms to explain zinc whisker growth have been proposed, but none of them are widely accepted and some are in conflict with each other. Most early investigations with regard to zinc whiskers studied 
their growth mechanisms. The first investigation was undertaken by Lindborg, who suggested that zinc whisker growth was driven by the internal macrostress of the zinc electrodeposit, which was formed in the deposit during electroplating. ${ }^{5,6}$ Sugiarto et al. later stated that whisker growth from bright zinc electrodeposits was induced by compressive stress that was derived from the brightener system, whilst dull zinc electrodeposits, without brighteners, did not develop whiskers. ${ }^{7}$ Baated et al. reported that Fe-Zn intermetallic compound (IMC) and zinc oxides were present at the interface between the zinc film and the steel substrate as well as within the electroplated zinc coatings themselves, both of which were able to produce compressive stresses within the zinc film and promote whisker growth. ${ }^{8,9}$ However, other investigators have reported that no intermetallic, or only a very thin layer of Fe-Zn IMC, was present at the interface, based on which they concluded that the development of Fe-Zn IMC was not associated with zinc whisker growth. ${ }^{10,11}$ Etienne et al. correlated the recrystallisation of zinc grains with whisker growth, reporting that grains near the root of whiskers were recrystallised, ${ }^{12,13}$ which is consistent with tin whisker growth mechanisms based on recrystallisation. ${ }^{14-16}$

A more thorough overview of zinc whisker-related research can be found in a recent review paper. ${ }^{17} \mathrm{In}$ comparison with tin whisker research, comparatively few papers have been published in relation to zinc whisker growth, and some of the results are contradictory, such as those relating to the influence of thermal treatments on whisker growth. ${ }^{7,18,19}$ The present work was undertaken to investigate the mechanism of whisker growth from zinc electrodeposits using scanning electron microscopy (SEM), dual beam focused ion beam scanning electron microscopy (FIBSEM), energy dispersive x-ray spectroscopy (EDS) and electron backscatter diffraction (EBSD). The presence of raised hemispherical features (nodules) on the deposit surface was found to be closely associated with $\mathrm{Zn}$ whisker growth, and the recrystallisation of $\mathrm{Zn}$ grains was responsible for whisker growth.

\section{EXPERIMENTAL}

Mild steel substrates (1.0 mm thickness) were electroplated with zinc using a proprietary bright alkaline cyanide-free zinc bath provided by MacDermid. The substrates, with dimensions $2 \times 4 \mathrm{~cm}$, were used, with no additional polishing or grinding operations, as cathodes, with an electroplated area of $2 \times 2 \mathrm{~cm}$. The anode material was a $\geq 99.9 \%$ zinc metal foil ( $0.3 \mathrm{~mm}$ thickness). Prior to deposition, the substrates were first washed using detergent, degreased using acetone, pickled in a $32 \mathrm{wt} . \%$ solution of [specific gravity (SG) 1.16] hydrochloric acid for $1 \mathrm{~min}$ to remove surface oxides, rinsed with deionised water and finally dried using hot air. The anode material underwent a similar pre-treatment, except that in the pickling stage it was immersed in a 20 vol.\% solution of (SG 1.83) sulfuric acid for $30 \mathrm{~s}$. All the electrodeposition was carried out at a cathode current density of $25 \mathrm{~mA} / \mathrm{cm}^{2}$ for $10 \mathrm{~min}$ and the resultant deposits exhibited an average thickness of approximately $6 \mu \mathrm{m}$. No agitation was applied during electrodeposition. After deposition, all the samples were stored at room temperature $\left(20^{\circ} \mathrm{C}\right)$.

SEM analysis of the surface morphology of alkaline non-cyanide zinc electrodeposits, and subsequent whisker growth from them, was carried out using a Carl Zeis (Leo) 1530 VP field emission gun scanning electron microscope (FEGSEM), equipped with an Oxford Instruments X-Max $80-\mathrm{mm}^{2}$ EDS detector. The accelerating voltage used for secondary electron imaging was $5 \mathrm{kV}$. The surface morphology was examined immediately after deposition. Whisker growth, in terms of growth morphologies, dimensions and densities, was initially monitored every week until the first whiskers were observed and then at monthly intervals.

The cross-sectional microstructure of the zinc electrodeposits and the whiskers growing from them were investigated using a FEI Nova 600 Nanolab dual beam focused ion beam (FIB). The ion beam energy used for both imaging and milling was $30 \mathrm{kV}$. Initially, a layer of platinum $(\sim 1 \mu \mathrm{m}$ thick $)$ was deposited on the area of interest for protection from ion milling. In order to observe the crosssection, a rectangular trench of approximately $40 \mu \mathrm{m}$ in length, $25 \mu \mathrm{m}$ in width and $10 \mu \mathrm{m}$ in depth was ion-milled using a current of $20 \mathrm{nA}$. After this, the area of interest went through two cleaning cross-section processes in sequence using currents of $7 \mathrm{nA}$ and $3 \mathrm{nA}$, respectively, to improve crosssection surface finish. The current used for ion beam imaging of the resultant cross-section was $30 \mathrm{pA}$.

Samples for electron backscatter diffraction (EBSD) analysis were prepared using a lift-out technique with the FEI Nova 600 Nanolab dual beam FIB. The lift-out procedure was as follows: the section to be analysed was partially cut free from the sample, using a current of $20 \mathrm{nA}$; only a bridge of $3 \mu \mathrm{m}$ in width was left, by which one side of the lift-out section remained attached to the sample. The other side of the section was then attached to an Omniprobe by platinum deposition, after which the bridge was cut and the section was completely free from the sample. Finally, the lift-out section was attached to a $\mathrm{Cu}$ grid using platinum deposition. An ultra-high speed Hikari EBSD camera system was used to investigate the crystallographic orientation, grain size and structure of the cross-section. For EBSD analysis, an accelerating voltage of $20 \mathrm{kV}$ and a step size of $0.05 \mu \mathrm{m}$ were used.

\section{RESULTS AND DISCUSSION}

\section{Investigation of Surface Morphology}

The surface morphology of $6 \mu \mathrm{m}$ alkaline noncyanide zinc electrodeposited on mild steel 

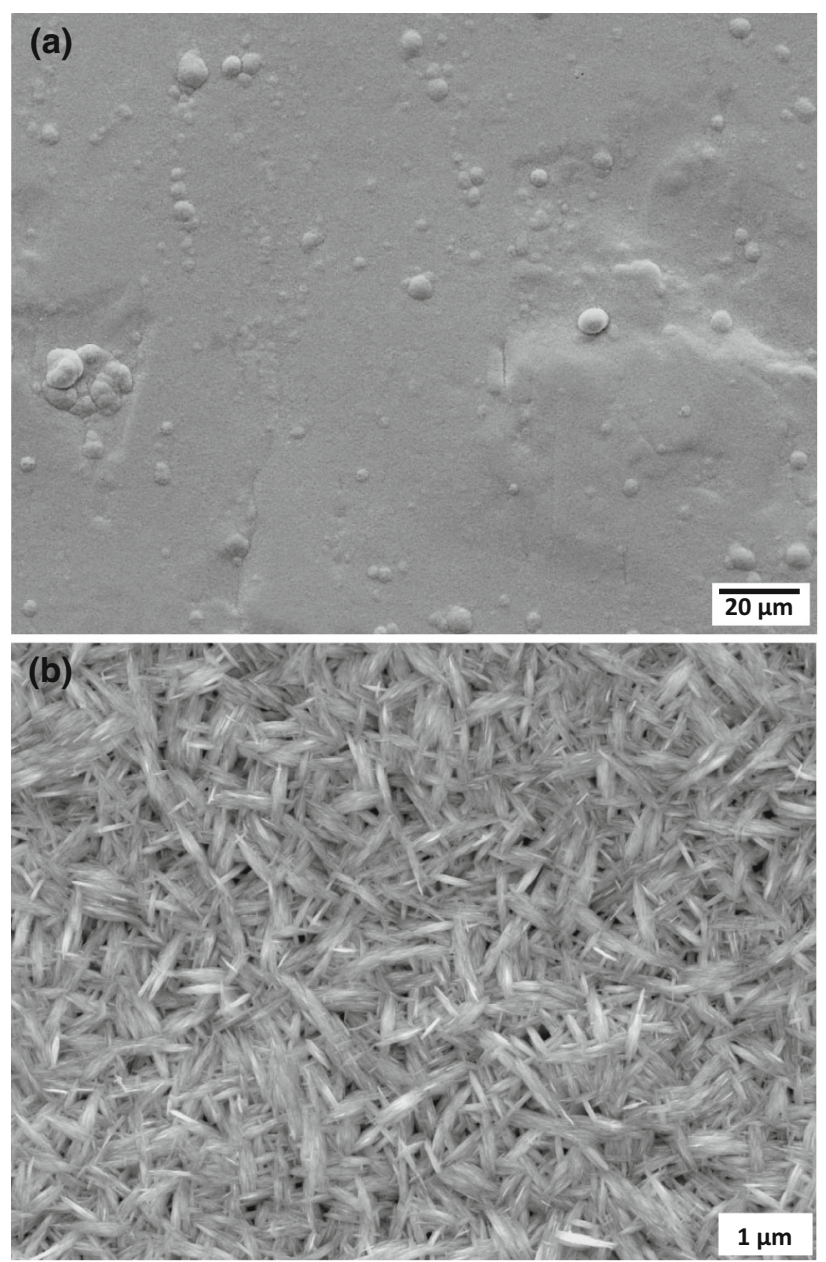

Fig. 1. SEM images showing the surface morphology of a $6-\mu \mathrm{m}$ alkaline non-cyanide zinc coating electrodeposited on mild steel at $25 \mathrm{~mA} / \mathrm{cm}^{2}$ : (a) low magnification and (b) high magnification.

substrates at $25 \mathrm{~mA} / \mathrm{cm}^{2}$ is shown in Fig. 1. SEM analysis immediately after deposition showed that a large number of raised hemispherical features were present on the deposit surface (Fig. 1a). These fea tures are referred to as "nodules". Higher-magnification imaging showed that the general surface was comprised of needle-like features of approximately $1 \mu \mathrm{m}$ in length and $100 \mathrm{~nm}$ in width (Fig. 1b). The needle-like features were randomly aligned over the deposit surface with no apparent preferred orientations. Similar structures have been reported in other published works for samples electrodeposited from other alkaline zinc plating baths. ${ }^{20,21} \mathrm{~A}$ higher magnification SEM image of one of the nodules is shown in Fig. 2. The nodules varied in size and some larger nodules were more than $20 \mu \mathrm{m}$ in diameter. Importantly, the nodules did not exhibit any typical whisker characteristics, such as striations, and were comprised of the same needle-like features as the remainder of the deposit; i.e. it can be concluded that the nodules were not any form of whisker eruption. Since SEM analysis was

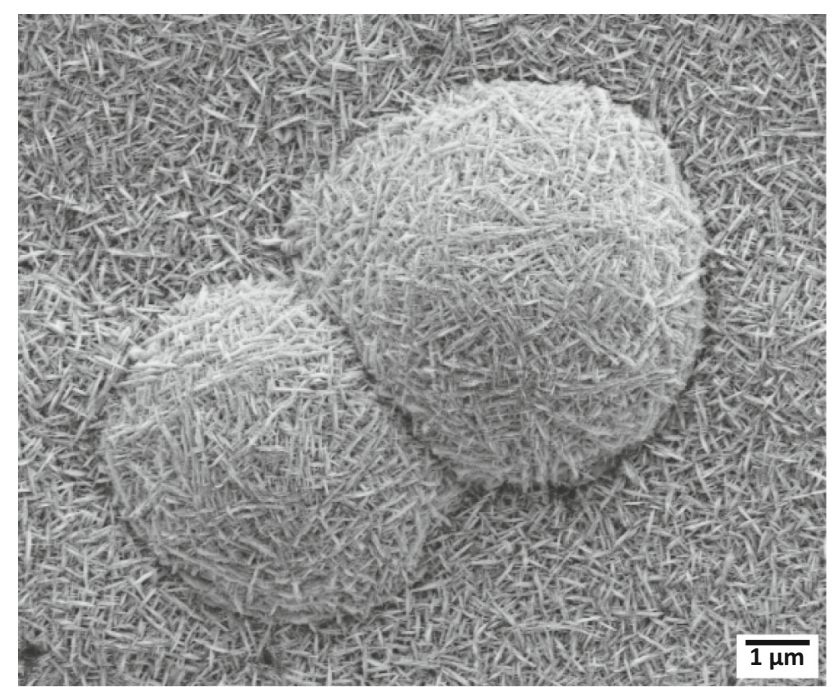

Fig. 2. SEM image showing a typical nodule present on the surface of a 6 - $\mu \mathrm{m}$ alkaline non-cyanide zinc coating electrodeposited on mild steel at $25 \mathrm{~mA} / \mathrm{cm}^{2}$.

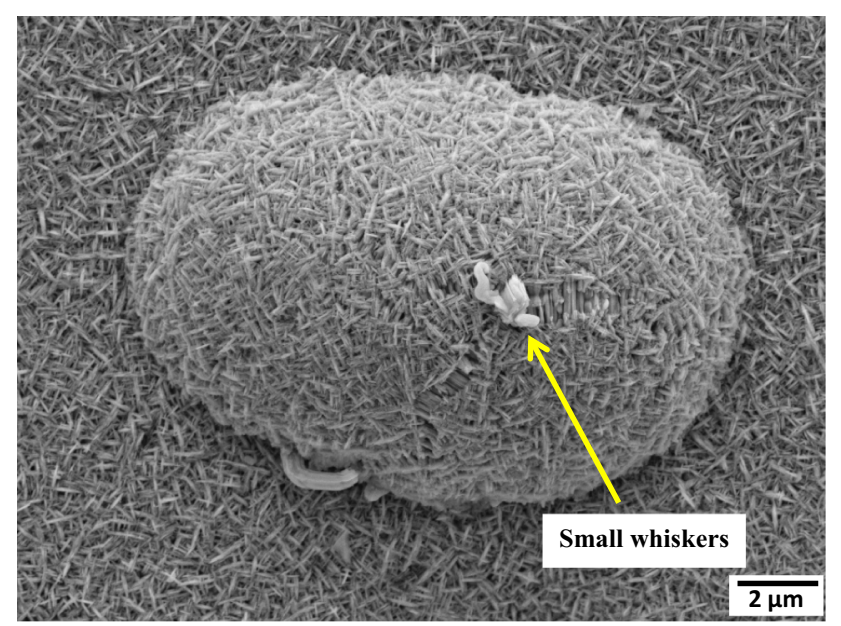

Fig. 3. SEM image showing an incipient whisker growth on the surface of a $6-\mu \mathrm{m}$ alkaline non-cyanide zinc coating electrodeposited on mild steel at $25 \mathrm{~mA} / \mathrm{cm}^{2} 23$ days after deposition.

conducted immediately after deposition and the nodules were already present, it is clear that the nodules were formed during electroplating rather than developing afterwards. This assumption is further supported by SEM analysis of the nodules after 6 months storage, which showed that the size and the shape of the nodules remained unchanged.

\section{Observation of Whisker Growth at Room Temperature}

SEM analysis showed that some incipient whiskers $\left(\sim 358\right.$ per $\left.\mathrm{cm}^{2}\right)$ (shown in Fig. 3), shorter than $1 \mu \mathrm{m}$ in length, were present on the samples 23 days after deposition. After 4 months storage, an increased number $\left(\sim 2176\right.$ per $\left.\mathrm{cm}^{2}\right)$ of whiskers, 

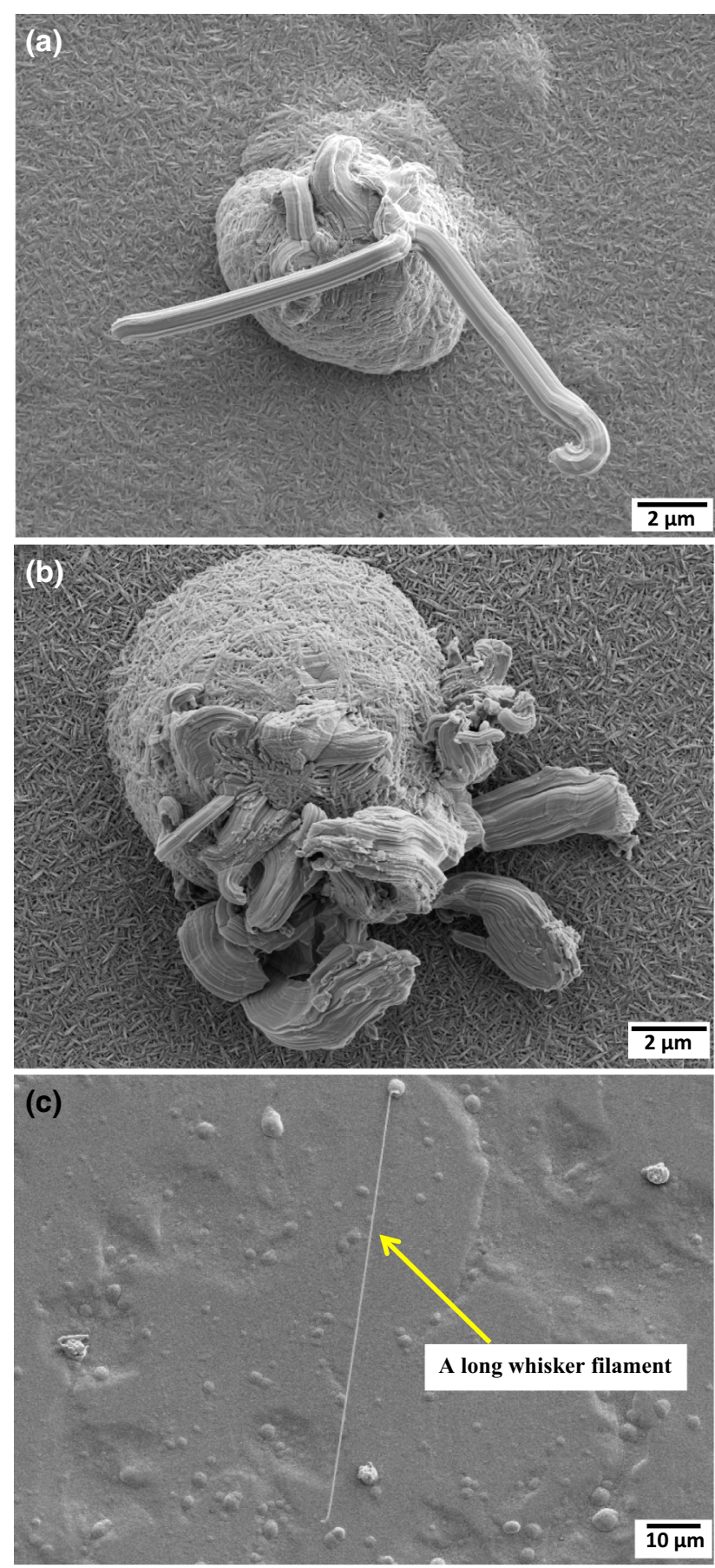

Fig. 4. SEM images showing whiskers growing associated with nodules on the surface of a $6-\mu \mathrm{m}$ alkaline non-cyanide zinc coating electrodeposited on mild steel at $25 \mathrm{~mA} / \mathrm{cm}^{2} 4$ months after deposition: (a) and (b) large irregular eruption-type whiskers and (c) a long filament-type whisker.

the majority of which were in the form of large irregular eruptions, were present on the deposit surface associated with the nodules. Examples of such eruption-type whiskers are shown in Fig. 4a and b. A few filament-type whiskers were also observed, some of which were greater than $300 \mu \mathrm{m}$
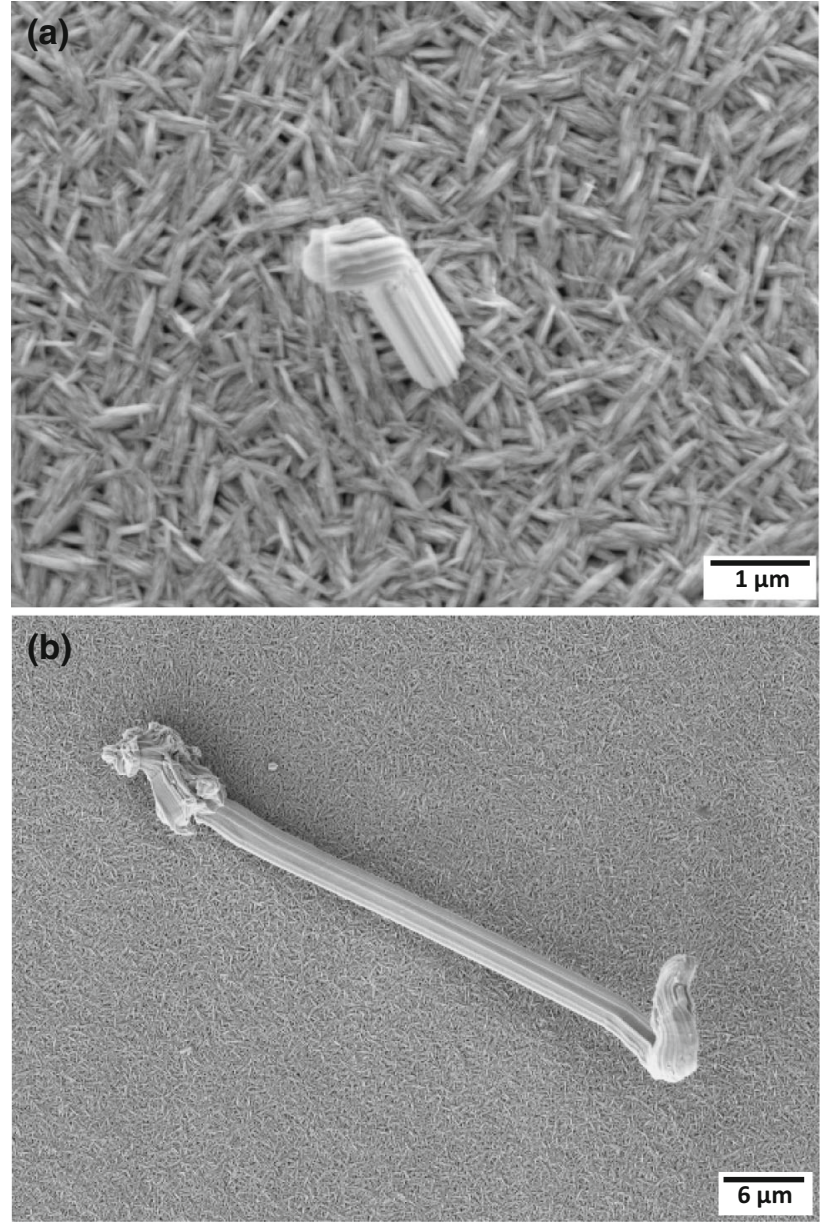

Fig. 5. SEM images showing whiskers growing from the planar deposit surface of a 6- $\mu \mathrm{m}$ alkaline non-cyanide zinc coating electrodeposited on mild steel at $25 \mathrm{~mA} / \mathrm{cm}^{2}$ : (a) a small whisker present 5 months after deposition and (b) a longer filament-type whisker present 10 months after deposition.

in length (Fig. 4c). These observations suggest that zinc electrodeposits on mild steel substrates prepared from alkaline cyanide-free zinc plating baths were prone to develop whiskers rapidly at room temperature. Importantly, it was observed that, up to 4 months storage, all the whisker growth was associated with the nodules. No whiskers were found growing from a planar region of the deposit. However, 5 months after deposition, a few small whiskers $\left(\sim 86\right.$ per $\left.\mathrm{cm}^{2}\right)$ were observed growing from a planar region of the deposit (Fig. 5a). Ten months after deposition, an increased number ( $\sim 599$ per $\mathrm{cm}^{2}$ ) of large whiskers growing from planar regions of the deposit were present (Fig. 5b). On the basis of these findings, whisker growth from planar regions of the deposit occurs much later than that associated with the nodules. It can therefore be deduced that the presence of the nodules on the surface was not a prerequisite for whisker growth, but promoted whisker growth by markedly shortening the incubation time. Therefore, the nodules must have some unique characteristics that are favourable for 
(a)

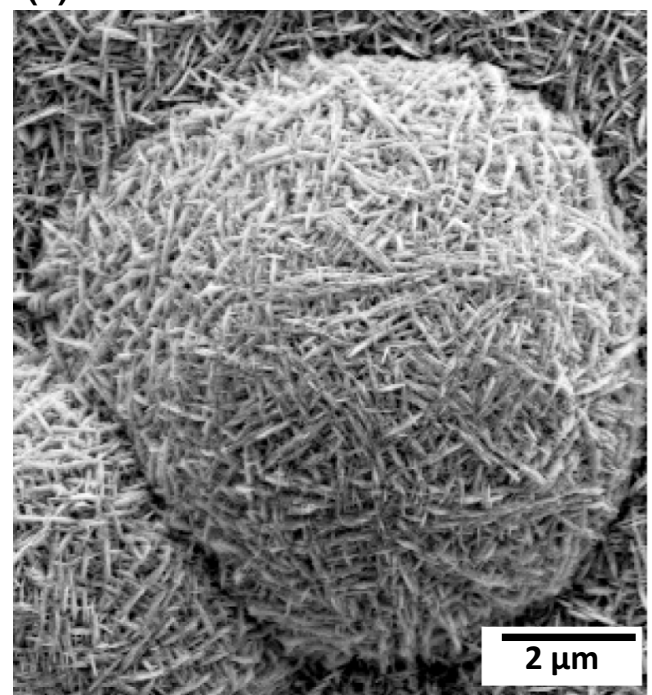

(c)

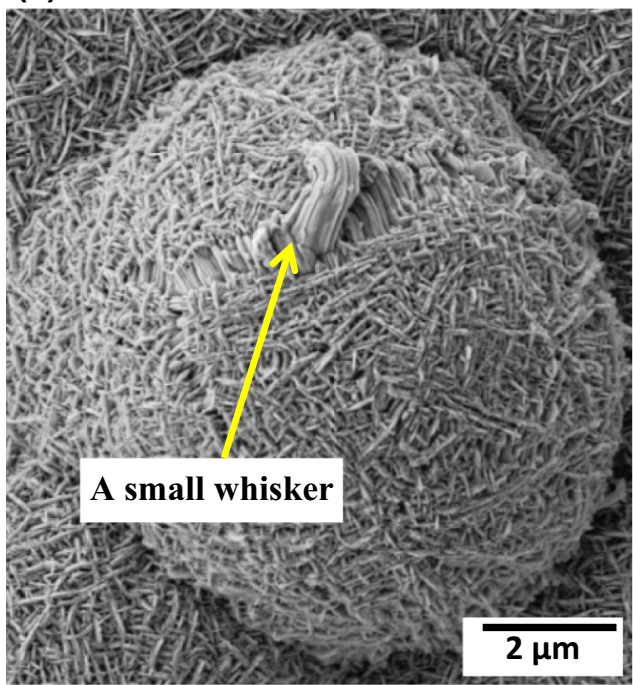

(b)

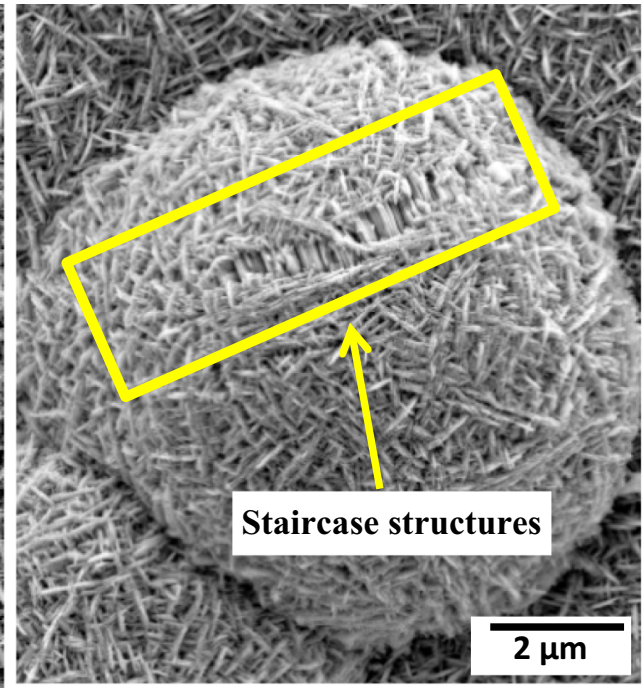

(d)

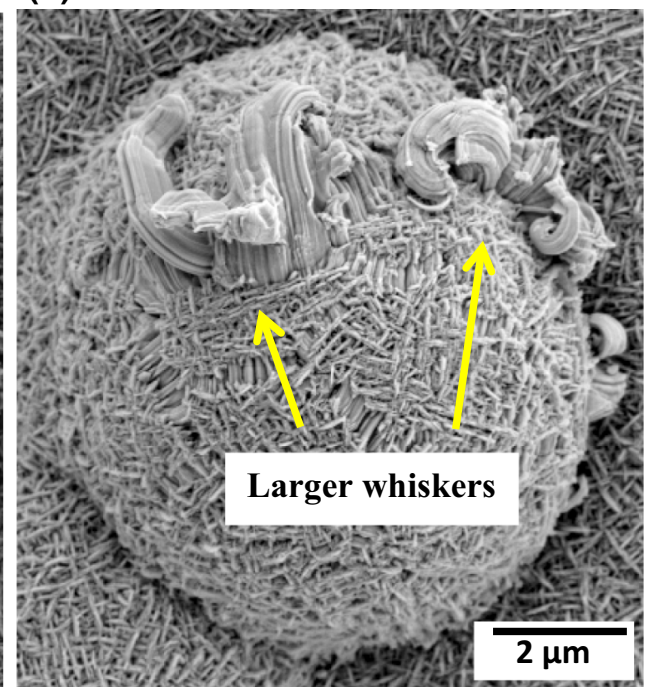

Fig. 6. SEM images showing the evolution of whiskers on a nodule on the surface of a $6-\mu \mathrm{m}$ zinc coating electrodeposited on mild steel at $25 \mathrm{~mA} / \mathrm{cm}^{2}$ as a function of storage time: (a) $2 \mathrm{~h}$ after deposition; (b) 29 days after deposition; (c) 44 days after deposition and (d) 68 days after deposition.

whisker formation compared with planar regions of the electrodeposit.

To understand the relationship between the nodules and whisker growth, three samples electrodeposited at $25 \mathrm{~mA} / \mathrm{cm}^{2}$ were prepared and five nodules on each sample were selected for periodic analysis in the SEM; an example of one such nodule is shown in Fig. 6. Changes to the surface morphology of the nodule were first observed 29 days after deposition (Fig. 6b). A series of parallel lines, which have the appearance of a "staircase", were present on the surface of the nodule. Such lines are henceforth termed "staircase structures". Forty-four days after deposition, a small whisker was observed growing from the top surface of the nodule in the region that had developed a staircase structure (Fig. 6c). SEM analysis 68 days after deposition showed that the original whisker had become larger and further whiskers had developed on the staircase structure (Fig. 6d). Also, further staircase structures had formed elsewhere on the nodule. It was observed that, within 1 month of deposition, staircase structures were present on the surface of most nodules. Once a staircase structure appeared on a nodule, subsequent whisker growth would typically follow. It was noted that whiskers only grew from the staircase structures rather than other regions on the nodules (Fig. 7a), i.e. it appears that a whisker was "extruded" from one or more steps of the staircase structures (Fig. 7b). SEM analysis after 10 months storage showed that all the nodules with staircase structures present had developed whiskers, whilst no whiskers were observed in the absence of staircase structures. It is suggested that 
(a)

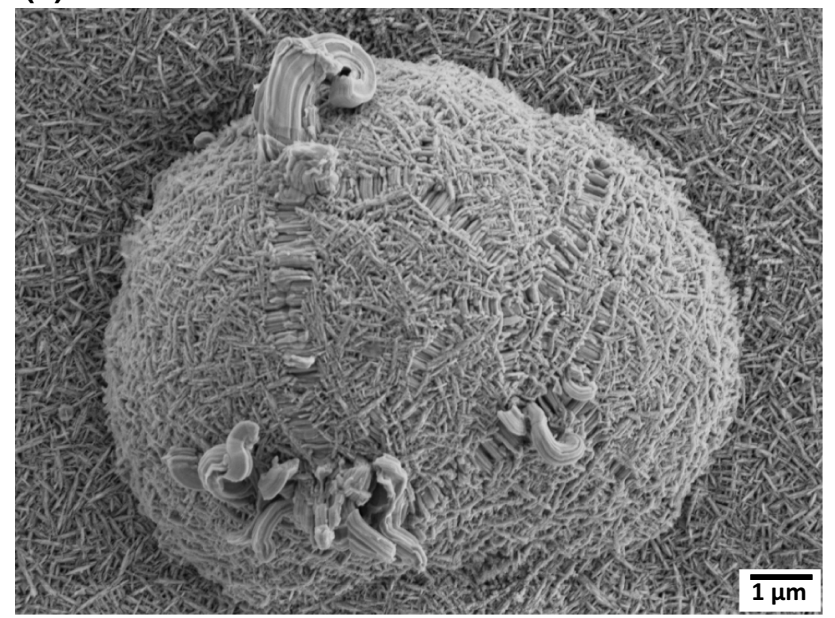

(b)

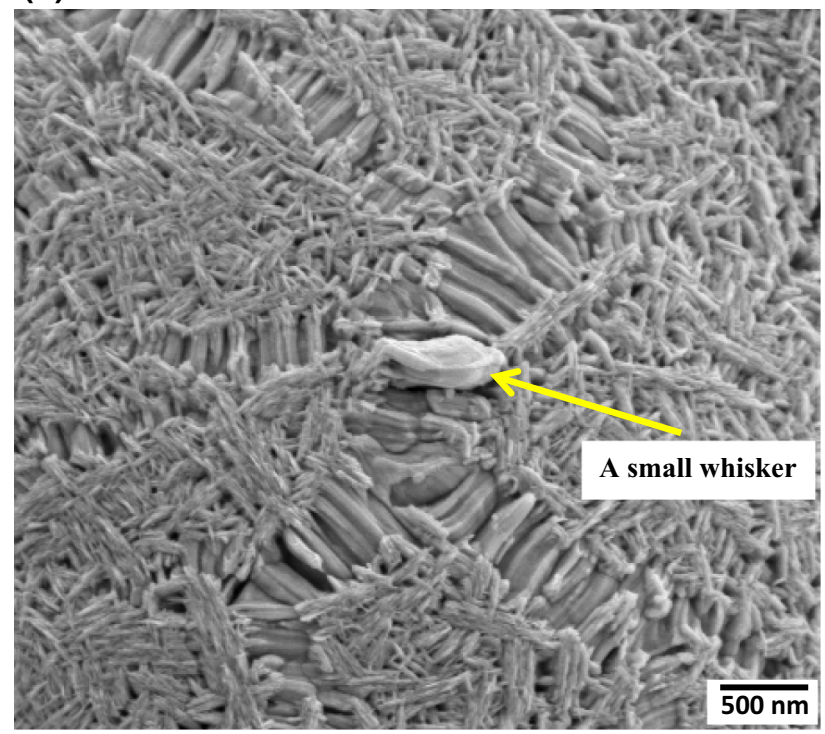

Fig. 7. SEM images showing whisker growth associated with the staircase structures on nodules on the surface of a $6-\mu \mathrm{m}$ zinc coating electrodeposited on mild steel at $25 \mathrm{~mA} / \mathrm{cm}^{2}$ : (a) whisker growth is always associated with the staircase structures and (b) a whisker "extrusion" from the staircase structures.

the staircase structures are pre-cursors to the growth of zinc whiskers associated with the nodules. Up to 10 months storage, no staircase structures were present on a planar region of the deposit. This may be the reason that the incubation time for whisker growth from a planar region of the deposit is considerably increased. All these observations suggest that the formations of nodules and the subsequent development of staircase structures are responsible for the shortened incubation time for whisker growth.

To further investigate the development of the staircase structures, periodic SEM analysis was carried out to monitor the change in whisker growth and the associated staircase structures on a nodule as a function of storage time. The first analysis was undertaken 5 weeks after deposition (Fig. 8a) and (a)

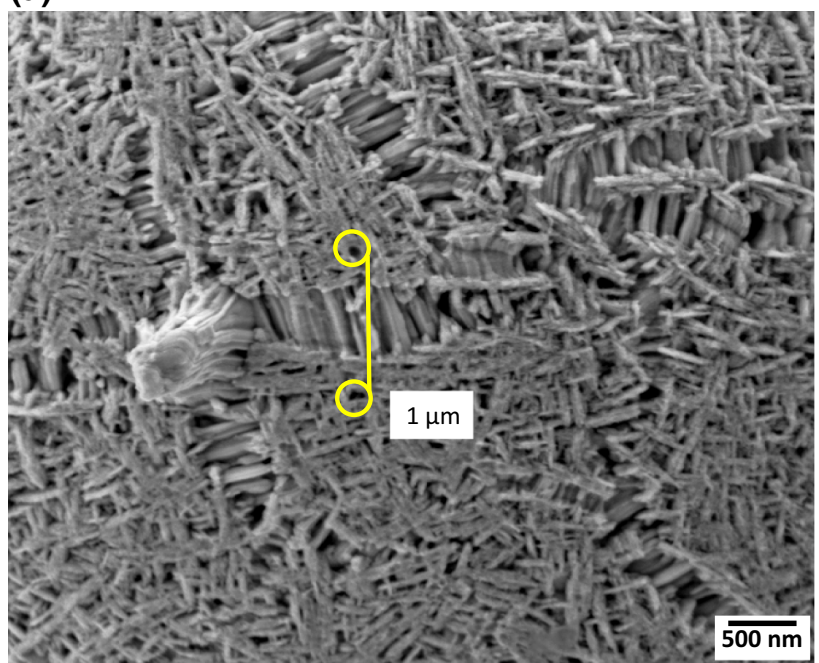

(b)

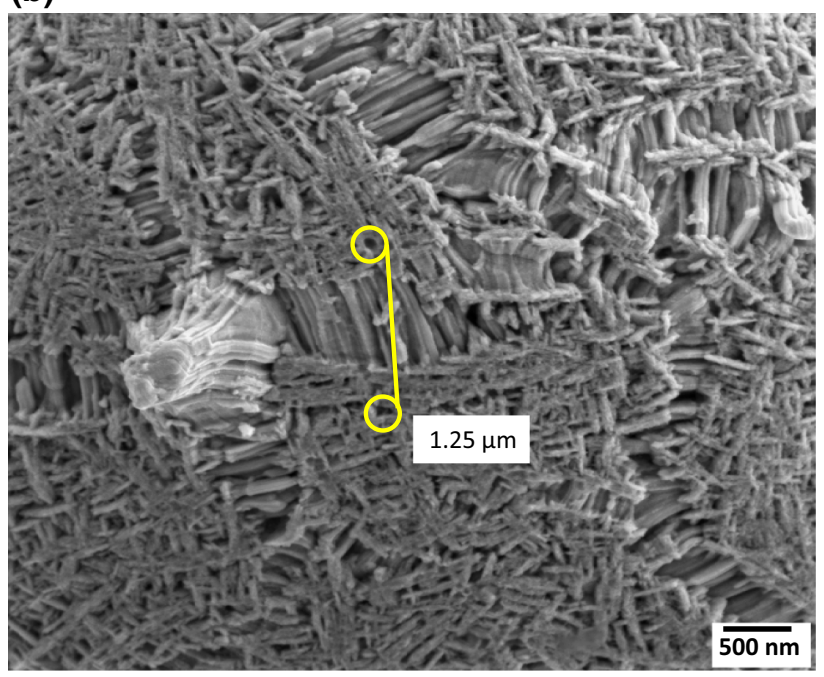

Fig. 8. SEM images showing whisker growth associated with the staircase structures on a nodule on the surface of a $6-\mu \mathrm{m}$ zinc coating electrodeposited on mild steel at $25 \mathrm{~mA} / \mathrm{cm}^{2}$ : (a) the first analysis undertaken 5 weeks after deposition and (b) the second analysis after a further 2 weeks.

the second analysis was after a further 2 weeks (Fig. 8b). On the second analysis, it was observed that not only had the whisker become slightly larger but also that the width of the staircase structure had increased, i.e. the staircase structures were developing along with whisker growth. Also, it was noted that two small pores on the right hand side of the whisker (circled in Fig. 8a and b) that were initially separated by a distance of $1 \mu \mathrm{m}$ had moved further apart to $1.25 \mu \mathrm{m}$ after 2 weeks further storage. On the basis of these findings, it is thought that the original surface of the nodule was expanded by the inclusion of new material, as the whisker and the staircase structure were growing. In other words, the as-deposited surface of the nodule itself was not transformed to create the increased width of the staircase structures. Additionally, from 


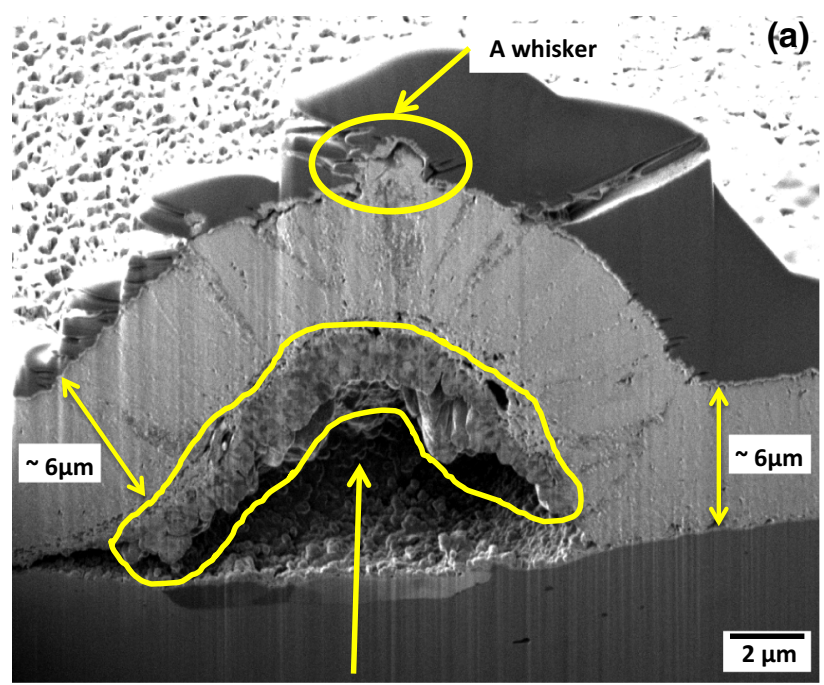

Dark grey areas showing a different contrast

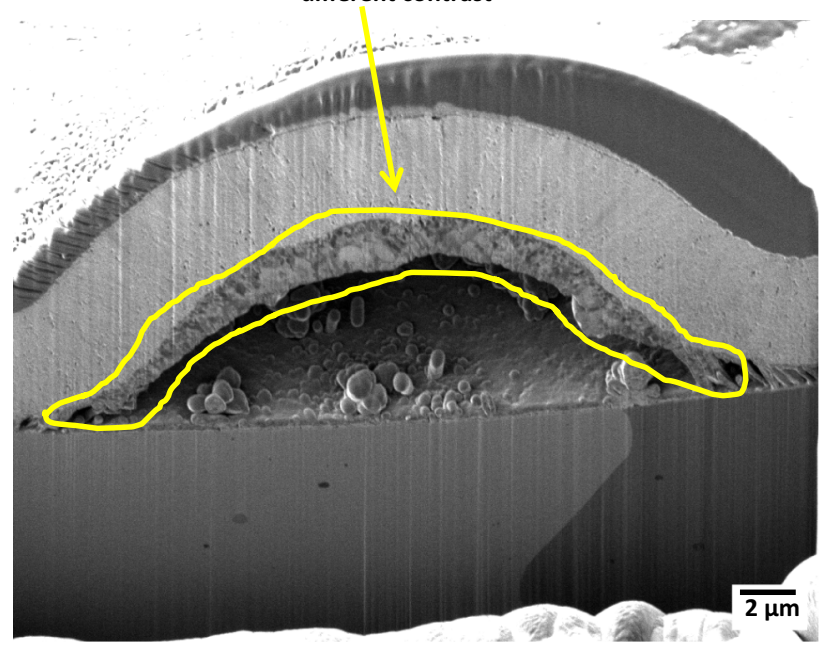

Fig. 9. FIB images showing the cross-section of nodules on the surface of a $6-\mu \mathrm{m}$ zinc coating electrodeposited on mild steel at $25 \mathrm{~mA} / \mathrm{cm}^{2}$ : (a) with whisker growth and (b) without whisker growth.

Fig. 6, it is apparent that, even though whiskers of significant size were formed on the nodule surface, which would have required a large volume of material in their formation, the apparent size of the nodule was not decreased. All these observations suggest that the nodule itself was not consumed by the whisker growth. It is speculated that the development of the staircase structures and subsequent whisker growth was probably supported by the long range diffusion of zinc.

\section{FIB Cross-section Analysis of Nodules}

Several nodules were cross-sectioned 4 months after deposition using a focused ion beam (FIB) technique. FIB analysis of nodules with and without whiskers growing from them is shown in Fig. 9a and $b$. In both cases, a cavity was present beneath both of the nodules. To date, a cavity has been observed beneath every cross-sectioned nodule (more than 20 nodules have been cross-sectioned), irrespective of whether or not a whisker is growing from it. It is noteworthy that the thickness of the deposit above a cavity was similar to that of the adjacent planar deposit and that the apparent increase in deposit thickness was solely due to the presence of the cavity beneath the nodule. FIB analysis of nodules on freshly electroplated samples showed that the cavities were already present; i.e. the nodules and the cavities were formed during deposition rather than developing afterwards. The mechanism for the development of the nodules and cavities was discussed in detail in a further zinc whisker paper published by the authors. ${ }^{22}$

The effect of nodule formation on zinc whisker growth has also been previously investigated. Fortier and Pecht ${ }^{11}$ found similar features on their freshly-prepared zinc electroplated steel samples. On the basis of EDS analysis, they reported that nodules contained a much higher level of oxygen compared with adjacent regions where nodules were not present and could be favourable sites for whisker growth. On the basis of their findings, EDX analysis was undertaken to determine whether the shortened incubation time for whisker growth from nodules in the current study could also be attributed to increased oxide formation on the nodule surface. The oxygen content and distribution in the regions containing nodules and the adjacent regions where nodules were not present was examined by EDX analysis, which indicated that there was no variation in oxygen levels. Both of the regions contained very little oxygen. These observations were not in agreement with Fortier and Pecht's investigations. However, EDX is not a very sensitive way to measure surface oxygen content, and thus it cannot be completely concluded that zinc oxidation was not associated with whisker growth from nodules.

\section{FIB Induced Artefacts}

From the FIB images, regions showing a different contrast to that of the normal deposit (dark grey areas arrowed in Fig. 9a and b) were always present on the inner surface of the cavities. TEM/EDS analysis, shown in Fig. 10, indicated that these regions were predominantly iron. The diffraction pattern obtained from such regions was comprised of diffuse rings, different from that of the substrate iron, which indicates that the iron in the dark grey areas was amorphous or nanocrystalline. These results suggest that the dark grey features were iron sputtered from the substrate during ion milling, which then re-deposited onto the inner surface of the nodules. Therefore, such features are an artefact of the FIB milling processes and not associated with either the electrodeposition process or whisker growth. 
Dark grey areas showing a different contrast
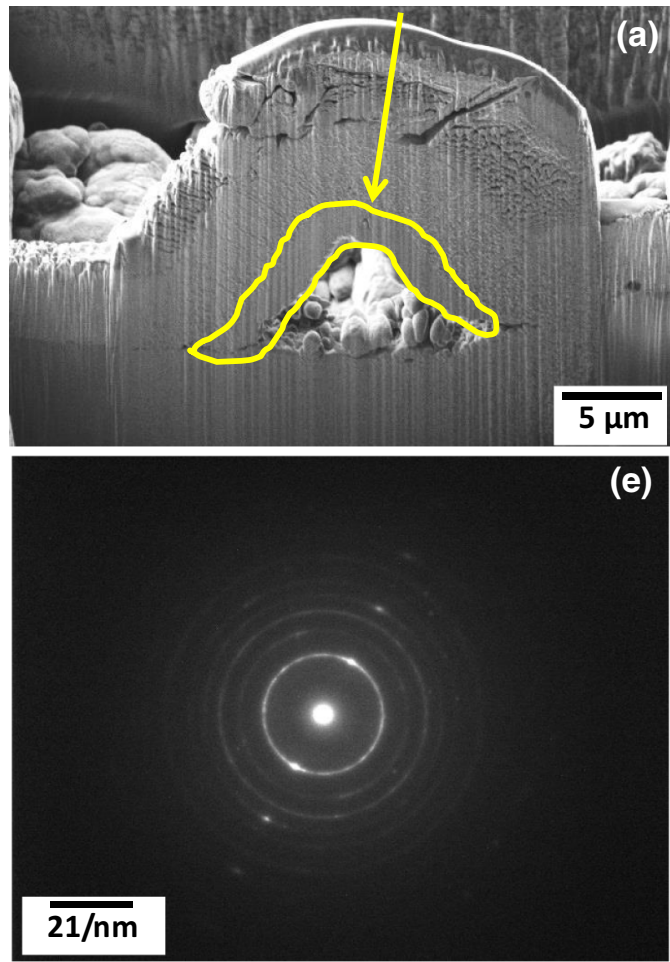

Dark grey areas showing a different contrast
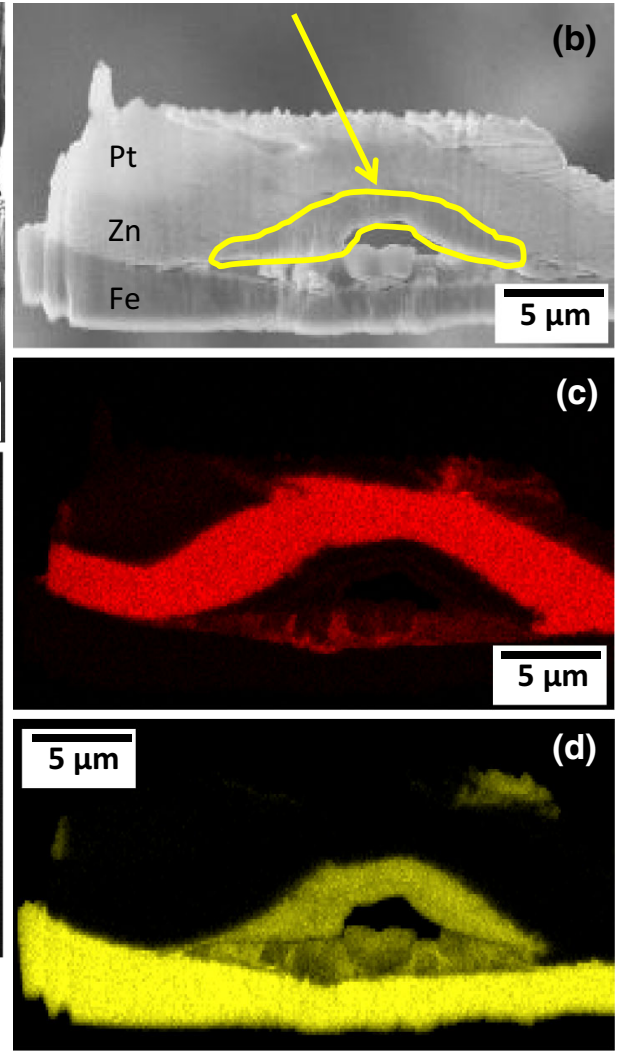

Fig. 10. TEM/EDS analysis of dark grey areas present on the inner surface of a nodule that was cross-sectioned from a 6- $\mu \mathrm{m}$ zinc coating electrodeposited on mild steel at $25 \mathrm{~mA} / \mathrm{cm}^{2}$ : (a) secondary electron beam image, (b) ion beam image, (c) Zn x-ray map, (d) Fe x-ray map and (e) TEM diffraction pattern obtained from the dark grey areas.

\section{Recrystallisation and Whisker Growth}

EBSD analysis of FIB lift-out sections has been used to investigate the cross-sectional microstructure of zinc coatings, nodules and whiskers. A sample, which was prepared 4 months after deposition, contained an eruption-type whisker growing from a nodule. The ion beam image and the crystallographic orientation map, shown in Fig. 11, indicated that the zinc deposit and part of the nodule was composed of fine grains of approximately $2 \mu \mathrm{m}$ in length and $200 \mathrm{~nm}$ in width. These grains were typically columnar with grain boundaries normal to the deposit surface. However, grains on the inner surface of the deposit at the apex of the nodule were equiaxed and much wider with a grain size of approximately $1 \mu \mathrm{m}$. The recrystallisation of these grains may be the first stage of the formation of whiskers. Furthermore, each whisker eruption was comprised of a single grain, the largest of which was around $7 \mu \mathrm{m}$ in width.

Another cross-section from the same sample was extracted 8 months after deposition. In this case, the cross-section contained a whisker growing from a planar region of the deposit where no nodules were present (Fig. 12). The EBSD results showed that grains near the whisker root were equiaxed, considerably larger than the typical grain size of the as-deposited columnar structure and exhibited a different crystallographic orientation to the unrecrystallised regions. Therefore, it is established that whisker growth is associated with recrystallisation, irrespective of whether whiskers were growing from a nodule or from a planar region of the deposit.

However, by only observing the microstructure after whisker growth, it cannot be established whether these recrystallised grains were already present immediately after deposition or formed after a certain incubation time. To more fully understand the point at which the recrystallized grains were formed, cross-sections were extracted and analysed from both a nodule and a planar region of a deposit straight after deposition. The crystallographic orientation map shown in Fig. 13 reveals that both the nodule and the planar region of the as-deposited coating were comprised of a fine columnar structure with no recrystallised grains evident. After a further 4 weeks storage, another cross-section was extracted from the same sample, 

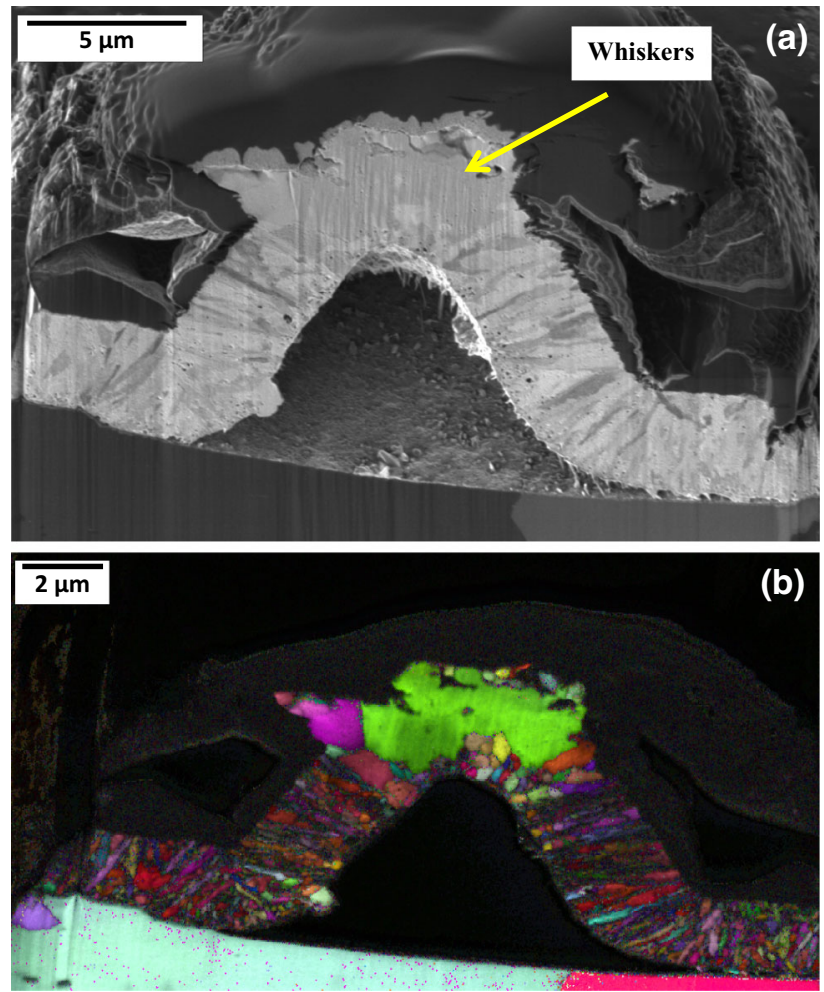

Fig. 11. EBSD analysis of a FIB lift-out section containing an eruption-type whisker growing from a nodule on the surface of a $6-\mu \mathrm{m}$ zinc coating electrodeposited on mild steel at $25 \mathrm{~mA} / \mathrm{cm}^{2}$ : (a) ion beam image and (b) crystallographic orientation map.

this time containing a nodule upon which staircase structures were present but no whisker formation had occurred (shown in Fig. 14a). It is suggested that the location of the recrystallised grains (indicated by an arrow in Fig. 14b) corresponds closely to the region on the surface where the staircase structures were present. Other regions of the nodule, where no staircase structures were present, retained their as-deposited columnar structure. From these results, it may be inferred that the staircase structures on the surface of the nodule are formed as a result of the recrystallisation of the asdeposited columnar grains. Given that subsequent whisker growth from the nodules occurs preferentially from the staircase structures, these observations clearly suggest that whisker growth is preceded by recrystallisation of the as-deposited columnar grains.

The relationship between recrystallisation and Sn whisker growth has been reported by Boguslavsky and Bush ${ }^{23}$ who hypothesised that the driving force for recrystallisation was the reduction of the interfacial energy of grain boundaries. During recrystallisation, certain grains continued to grow at the expense of the adjacent grains, and thus the number of grain boundaries was decreased and the total surface energy was lowered. Since zinc metal can readily recrystallise at room temperature $\left(25^{\circ} \mathrm{C}\right)^{24}$ and zinc electrodeposits comprising of a columnar
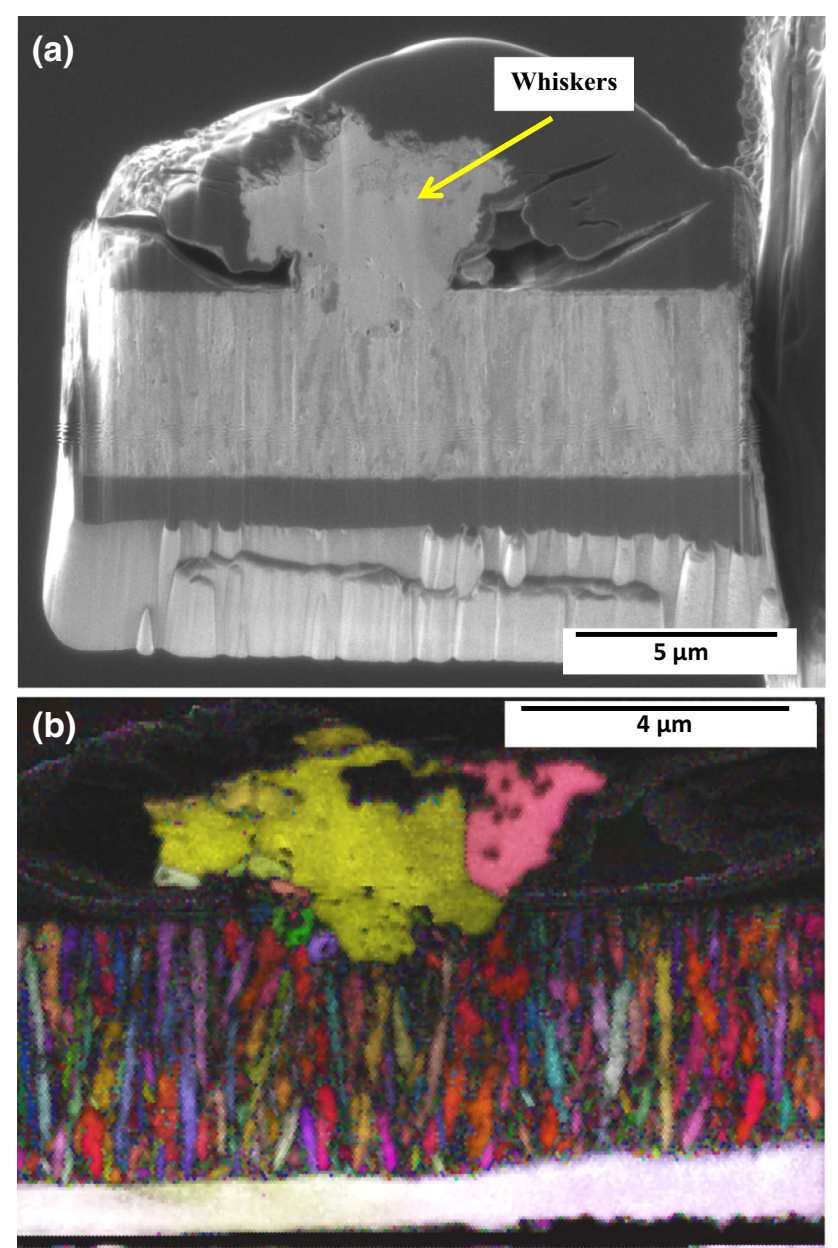

Fig. 12. EBSD analysis of a FIB lift-out section containing an eruption-type whisker growing from the flat deposit surface of a 6- $\mu \mathrm{m}$ zinc coating electrodeposited on mild steel at $25 \mathrm{~mA} / \mathrm{cm}^{2}$ : (a) ion beam image and (b) crystallographic orientation map.

structure have been shown previously to be under compressive stresses, ${ }^{9,13}$ the recrystallisation process can occur spontaneously at room temperature to produce a strain-free structure. Boguslavsky and Bush suggested that whisker growth was, in essence, a form of abnormal grain growth; however, not all the recrystallised grains subsequently grew into whiskers, which means that those grains that grew as whiskers must have some unique characteristics compared with other grains. They summarised that grains with different crystallographic orientations will have different surface energies and those having a low surface energy will grow preferentially to relieve the stress. However, they did not provide detailed information with regard to the energetically favourable orientations for whisker growth.

In the current study, the time dependent recrystallisation of the as-deposited columnar structure is thought to be associated with the difference in the incubation time between whisker growth from 

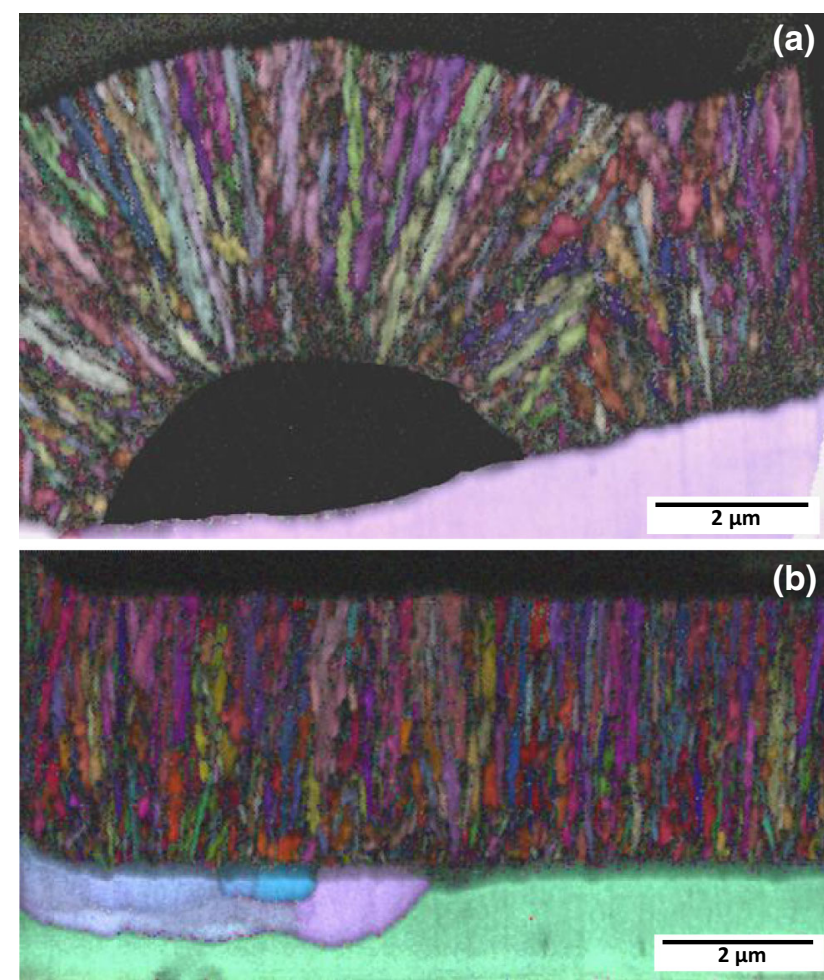

Fig. 13. EBSD analysis, immediately after deposition, of a FIB liftout section containing (a) a nodule on the surface of a $6-\mu \mathrm{m}$ zinc coating electrodeposited on mild steel at $25 \mathrm{~mA} / \mathrm{cm}^{2}$ and (b) the flat deposit surface of a $6-\mu \mathrm{m}$ zinc coating electrodeposited on mild steel at $25 \mathrm{~mA} / \mathrm{cm}^{2}$.

nodules and that from a planar region of the deposit. In the as-deposited condition, due to their geometry, the nodules were subjected to a larger stress compared with the planar deposit, which is favourable for recrystallisation to occur to relieve the stress; i.e. recrystallisation of the columnar structure occurs more rapidly within the nodules. This assumption was supported by the EBSD results (Figs. 11 and 12), which showed that, for whisker growth associated with nodules, in addition to the whisker grain, a significant number of other recrystallised grains were present near the whisker root after 4 months storage, while for whisker growth from a planar region of the deposit, within 8 months of deposition no other recrystallised grains were present near the whisker root except those that grew as whiskers. It is suggested that the presence of the nodules accelerates the recrystallisation of the as-deposited columnar structure, and hence shortens the incubation time for whisker growth.

\section{Zinc Whisker Growth into Cavities}

It was found that a number of large features were growing into the cavities beneath the nodules (Fig. 15). The features have the appearance of curved whisker eruptions and show a typical whisker morphology, e.g. striations are present on
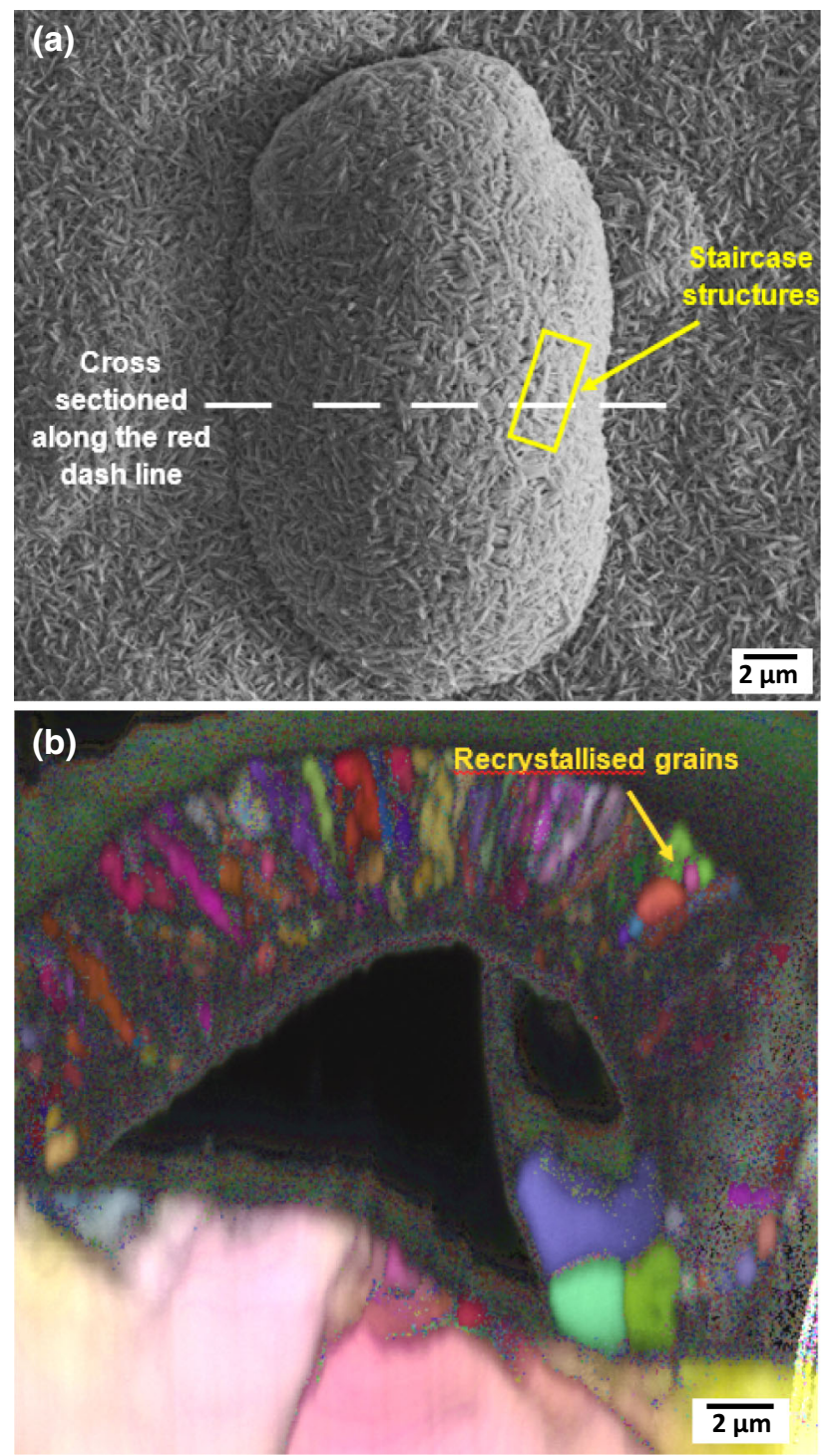

Fig. 14. EBSD analysis, 4 weeks after deposition, of a FIB lift-out section containing a nodule on the surface of a $6-\mu \mathrm{m}$ zinc coating electrodeposited on mild steel at $25 \mathrm{~mA} / \mathrm{cm}^{2}$ : (a) electron beam image and (b) crystallographic orientation map.

their surface. EDS and EBSD analysis of one such feature, shown in Fig. 16, indicated that it was composed of pure zinc with an increased grain size. Also, a long chain of recrystallised grains was observed linking the feature to the outer surface of the deposit. The size of the recrystallised grains increases as they approach the root of the feature at the inner surface of the cavity. The feature itself was formed by the growth of a single recrystallised grain. All these observations suggest that the feature was formed as a result of recrystallisation; i.e. the growth mechanism is similar to that which results in whisker growth from the external surface of nodules and the planar deposit. Hence, these features were thought to be zinc whiskers growing downwards into the cavity beneath the nodules. 


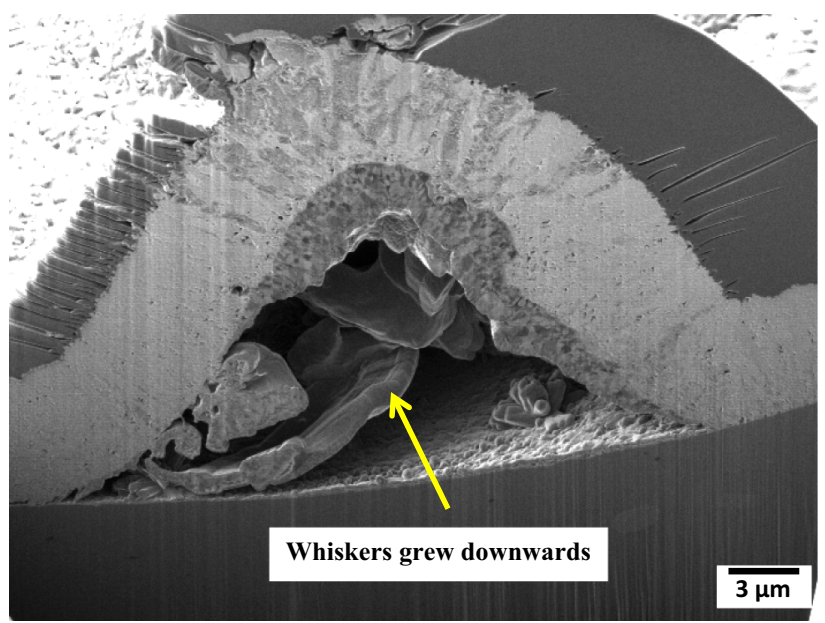

Fig. 15. FIB image showing a large feature growing into a cavity beneath a nodule on the surface of a $6-\mu \mathrm{m}$ zinc coating electrodeposited on mild steel at $25 \mathrm{~mA} / \mathrm{cm}^{2}$.

\section{The Effect of Fe-Zn Intermetallic Compounds on Whisker Growth}

Since it is well known that the presence of $\mathrm{Cu}-\mathrm{Sn}$ intermetallic compounds is closely associated with whisker growth from tin electrodeposits on copper substrates, ${ }^{25,26}$ the formation of $\mathrm{Fe}-\mathrm{Zn}$ intermetallic compounds and their possible role in zinc whisker growth has been investigated. Three cross-sections were extracted using a FIB lift-out technique from the surface of a 10-month old sample with whisker eruptions growing from a planar region of the deposit. Both ion beam and secondary electron beam imaging were used to observe the $\mathrm{Fe} / \mathrm{Zn}$ interface, shown in Fig. 17. On the basis of this analysis, there is no obvious evidence of $\mathrm{Fe}-\mathrm{Zn}$ intermetallic formation at the interface or within the zinc coating beneath the whiskers. Whisker growth from zinc electroplated steel does not appear to be associated with the formation of Fe-Zn intermetallic compounds. However,
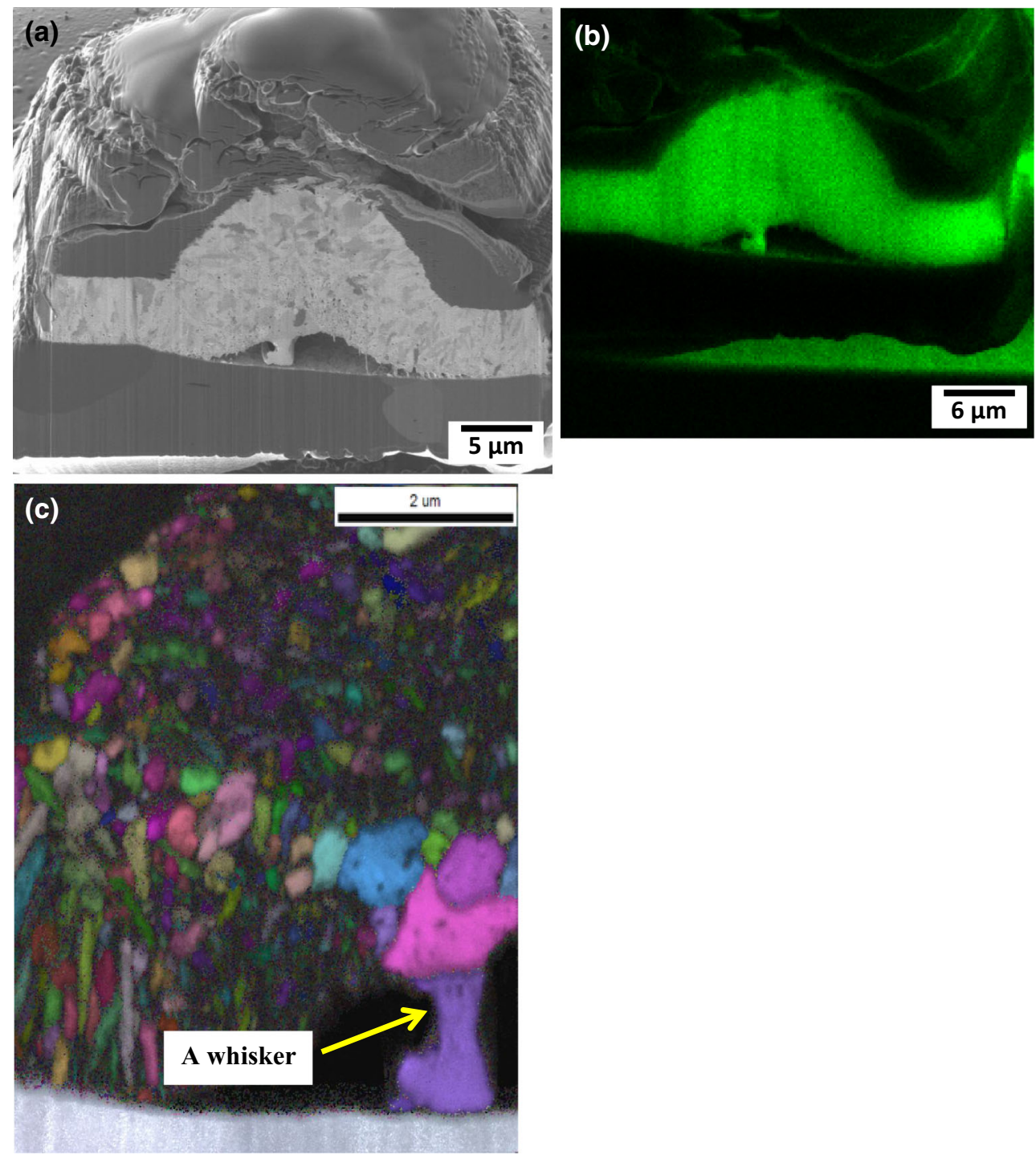

Fig. 16. EDS and EBSD analysis of a feature growing into a cavity beneath a nodule on the surface of a 6 - $\mu \mathrm{m}$ zinc coating electrodeposited on mild steel at $25 \mathrm{~mA} / \mathrm{cm}^{2}$ : (a) ion beam image, (b) Zn x-ray map (c) crystallographic orientation map. 


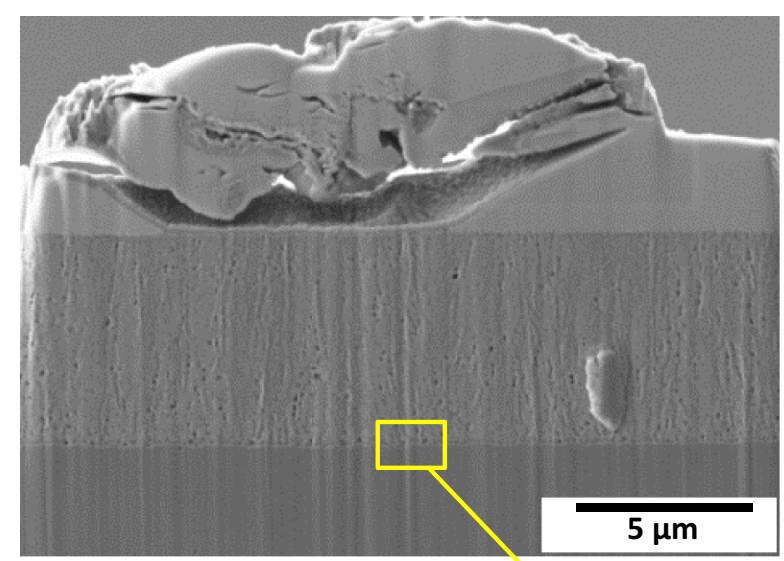

(a)

(b)
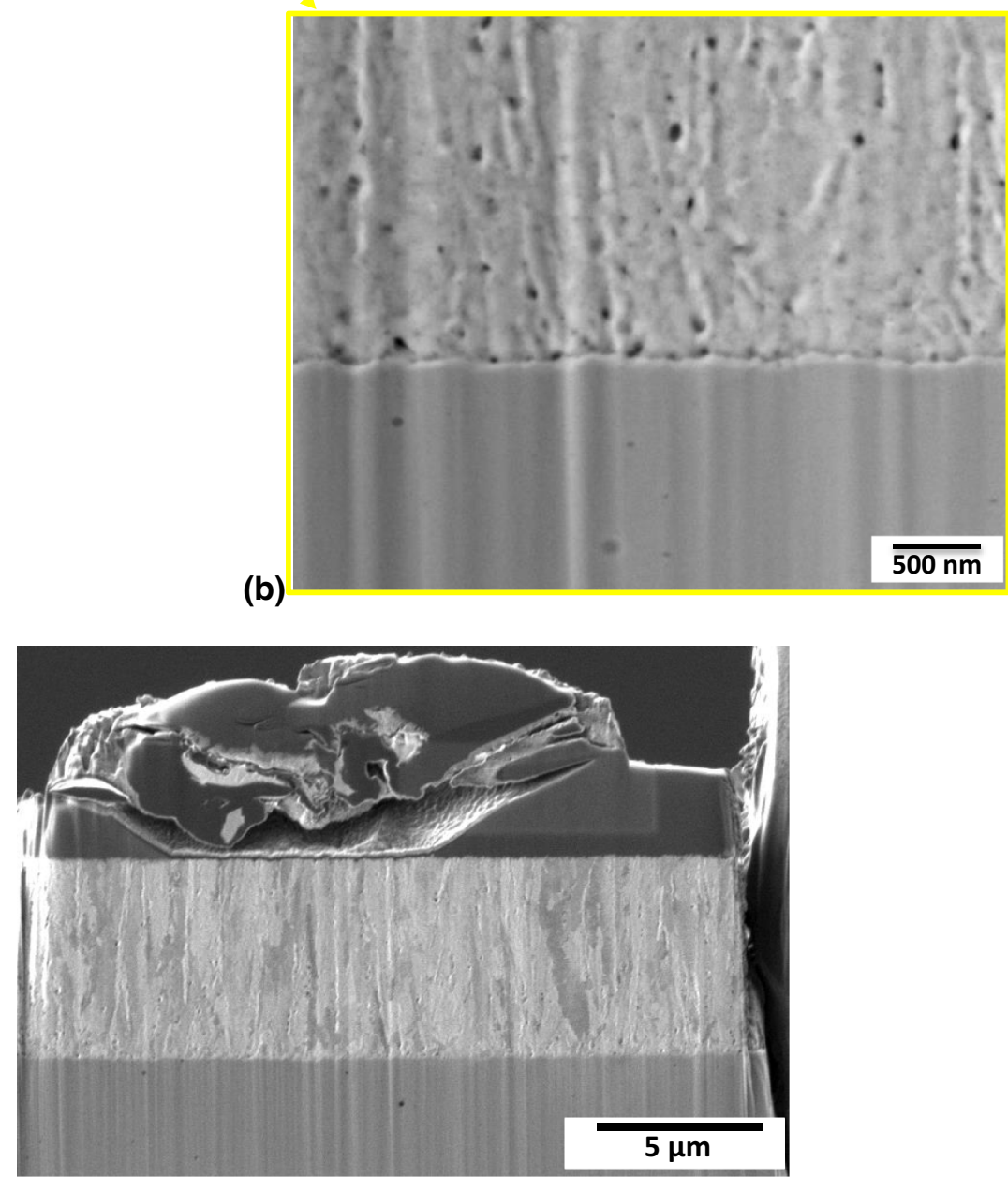

(c)

Fig. 17. The cross-sectional microstructure of a $6-\mu \mathrm{m}$ zinc coating electrodeposited on mild steel at $25 \mathrm{~mA} / \mathrm{cm}^{2}$ : (a) low magnification SEM image, (b) high magnification SEM image at the Fe/Zn interface and (c) ion beam image.

it must be remembered that these observations were made within 10 months of electroplating. Whether $\mathrm{Fe}-\mathrm{Zn}$ intermetallic compound growth become apparent at extended time intervals is not known. Baated et al. ${ }^{8,9}$ reported that Fe-Zn intermetallic compounds were not only formed at the $\mathrm{Fe} /$ $\mathrm{Zn}$ interface but also within the electroplated zinc coatings on a specimen obtained from a computer room floor tile that has been in service for more than 10 years. They concluded that the presence of $\mathrm{Fe}-\mathrm{Zn}$ intermetallic compounds could result in the development of compressive stresses within the zinc film, and thus the subsequent growth of whiskers from the surface to relieve the stress. 
Interestingly, although no Fe-Zn intermetallic compounds were present, a large number of voids were observed within the zinc deposit (Fig. 17b). It is not known whether the presence of these voids is associated with zinc whisker growth. Fortier and Pecht ${ }^{11}$ also observed voids in the deposit beneath zinc whiskers and proposed that whisker growth was associated with the oxidation of zinc metal that occurred on the deposit surface. They rationalised that the oxidation process produced voids near the surface, which then diffused downwards into the zinc film. The inward diffusion of the voids caused zinc atoms to migrate upwards to the surface and form whiskers. In the current study, voids were not only formed in the deposit beneath whiskers but also randomly distributed throughout the entire coating layer, which may indicate that void formation was not uniquely associated with whisker growth. It is possible that these voids were generated by dissolved hydrogen incorporated into the deposit during deposition, due to the low cathode current efficiency (approximately 30-40\%) of alkaline zinc electrodeposition.

\section{CONCLUSIONS}

Zinc electrodeposits on mild steel substrates prepared from alkaline cyanide-free zinc electroplating baths were prone to develop whiskers rapidly at room temperature. A number of long filament-type whiskers ( $>300 \mu \mathrm{m}$ in length) were present within 4 months of deposition.

A large number of raised hemispherical nodules were present on the deposit surface immediately after zinc deposition. The incubation time for whisker growth from the nodules was considerably reduced from $\sim 5$ months to $\sim 1$ month compared with that from the planar deposit surface. Staircase structures were found forming on the surface the nodules, which were pre-cursors to the growth of zinc whiskers associated with the nodules.

EBSD analysis of the cross-sectional microstructure of zinc coatings, nodules and whiskers indicated that the zinc deposit and the majority of the nodules were composed of fine columnar grains. However, recrystallisation of the as-deposited columnar structure occurred at the whisker root, irrespective of whether whiskers were growing from a nodule or from a planar region of the deposit.

Both secondary electron and ion beam images showed no evidence of Fe-Zn intermetallic formation at the $\mathrm{Fe} / \mathrm{Zn}$ interface or within the zinc coating beneath whiskers. It is suggested that, for the alkaline zinc electroplating investigated in this study, Fe-Zn intermetallic compounds are not associated with whisker growth.

\section{ACKNOWLEDGEMENTS}

The authors would like to thank the United Kingdom Engineering and Physical Sciences Research Council (EPSRC) Innovative Electronics
Manufacturing Research Centre for funding this research through the WHISKERMIT programme at Loughborough University.

\section{OPEN ACCESS}

This article is distributed under the terms of the Creative Commons Attribution 4.0 International License (http://creativecommons.org/licenses/by/4.0/), which permits unrestricted use, distribution, and reproduction in any medium, provided you give appropriate credit to the original author(s) and the source, provide a link to the Creative Commons license, and indicate if changes were made.

\section{REFERENCES}

1. M. Sampson and H. Leidecker, Basic Information Regarding Tin Whiskers, (NASA 2009), http://nepp.nasa.gov/ whisker/background/index.htm. Accessed 09 Feb 2013.

2. H.L. Cobb, Mon. Rev. Am. Electroplat. Soc. 33, 28 (1946).

3. S. Tucker, The Case of the "Zinc Whiskers", (Canada'S Health-care Newspaper 2003), http://www.hospitalnews. com/the-case-of-the-zinc-whiskers/, Accessed 10 Feb 2013.

4. A. Svaldi, Zinc "whiskers" cause computer outages in Colorado state offices, (Knight Ridder/Tribune Bus News 2004), http://www.highbeam.com/doc/1G1-118857697.html, Accessed 10 Feb 2013.

5. U. Lindborg, Acta Metall. 24, 181 (1976)

6. U. Lindborg, Metall. Trans. A 6A, 1581 (1975).

7. H. Sugiarto, I.R. Christie, and B.P. Richards, Trans. Inst. Met. Finish. 62, 92 (1984).

8. A. Baated, K.S. Kim, and K. Suganuma, in International Conference on Packaging Technology High Density Packaging (2009).

9. A. Baated, K.S. Kim, and K. Suganuma, J. Mater. Res. 25, 2175 (2010).

10. H.L. Reynolds and R. Hilty, in Investigations of Zinc Whiskers using FIB Technology IPC/JEDEC Lead-Free North America Conference (2004), p. 1.

11. A. Fortier and M.G. Pecht, IEEE Trans. Compon. Packag. Manuf. Technol. 2, 739 (2012).

12. A. Etienne, E. Cadel, A. Lina, L. Cretinon, and P. Pareige, IEEE Trans. Compon. Packag. Manuf. Technol. 2, 1928 (2012).

13. A. Etienne, E. Cadel, A. Lina, L. Cretinon, and P. Pareige, J. Electr. Mater. 42, 272 (2012).

14. W.C. Ellis, Trans. Met. Soc. AIME 236, 872 (1966).

15. N. Furuta and K. Hamamura, Jap. J. App. Phys. 9, 1404 (1969).

16. J. Smetana, IEEE Trans. Electr. Packag. Manuf. 30, 11 (2007).

17. L. Wu, M.A. Ashworth, and G.D. Wilcox, Trans. Inst. Met. Finish. 93, 1 (2015).

18. R. Chapaneri, A.J. Ebbage, G.D. Wilcox, G.W. Critchlow, A. Chojnicki, T. Pearson, and A.J. Rowan, Trans. Inst. Met. Finish. 87, 159 (2009).

19. H.R. Garner, D. Dickie, and C.W. Skimin, Whisker Growth from Decorative Zinc Electrodeposits, A Working Paper of the Harshaw Chemical Company, Cleveland, OH, USA, 1991.

20. M. Wery, J.C. Catonne, and J.Y. Hihn, J. Appl. Electron. $30,165(2000)$.

21. J.C. Hsieh, C.C. Hu, and T.C. Lee, Surf. Coatings Technol. 203, 111 (2009)

22. L. Wu, M. Ashworth, and G. Wilcox, ECS Trans. 66, 1 (2015).

23. I. Boguslavsky and P. Bush, in Tin, APEx Conference (2003). 
Wu, Ashworth, and Wilcox

24. N. Luptáková, M. Benák, L. Hajduchová, and F. Pešlová, Microstructure Analysis of Zinc and Zinc Alloys (Materials Engineer, 2012). http://www.materialing.com/zinc_alloys. Accessed 08 June 2014.
25. K. Fujiwara, M. Ohtani, T. Isu, and S. Nango, Thin Solid Films 70, 153 (1980).

26. K.N. Tu and R.D. Thompson, Acta Metall. 30, 947 (1982). 